\title{
The associations between phosphatidylethanolamine N-methyltransferase gene, dietary choline intake and anxiety and depression in healthy UK adults
}

\author{
K. Harrison ${ }^{1}$, C. Foster-Brown ${ }^{1}$, C. Kelaiditis ${ }^{2}$, Y. Mavrommatis ${ }^{1}$ and L. Pilic ${ }^{1}$ \\ ${ }^{1}$ Faculty of Sport, Health and Applied Science, St Mary's University Twickenham, Strawberry Hill, TWI 4SX and \\ ${ }^{2}$ Department of Life Sciences, University of Roehampton, London SW15 5 PU
}

Mental illness presents a serious public health concern worldwide. In England, one in six adults met the criteria for a common mental disorder (CMD) such as anxiety or depression in 2014. Moreover, women were more likely to experience CMD symptoms than men $^{(1)}$. Diet has been identified as a modifiable risk factor for depression and anxiety and may serve as a strategy for successful prevention of such disorders ${ }^{(2)}$. Low levels of choline, which acts as a precursor for the neurotransmitter acetylcholine and can be obtained from foods such as eggs or liver, may be associated with anxiety and depression ${ }^{(3)}$. Endogenous choline synthesis depends on the activity of the Phosphatidylethanolamine N-methyltransferase (PEMT) gene which may be determined by the rs4646343 variant ${ }^{(4)}$. It is hypothesised that carriers of the $\mathrm{T}$ risk allele may require larger amounts of dietary choline to compensate for lower $P E M T$ activity and choline levels. However, research on the topic is scarce, especially in the UK, where no dietary recommendations for this essential nutrient are established. The aim of this study was to explore the associations between PEMT rs4646343, dietary choline and anxiety and depression.

Forty-five healthy UK adults (15 male and 30 female, mean age $33 \pm 7.8$ years and body mass index $23.3 \pm 2.9 \mathrm{~kg} / \mathrm{m} 2)$ took part in this cross-sectional study. Genomic DNA was extracted from saliva and participants were genotyped for the PEMT rs4646343. Three-day food diaries were collected via Libro app (Nutritics LTD, Ireland). Choline intake was quantified using the United States Department of Agriculture food composition database considering it is not a part of the UK food composition tables. Anxiety and depression scores were calculated with the validated Hospital Anxiety and Depression Scale (HADS) questionnaire. Overall questionnaire scores were included as continuous variables in the analyses. Participants were also categorised as normal $(45 \%)$, borderline $(22 \%)$ or abnormal $(33 \%)$ cases for anxiety and depression. Study was approved by the St Mary's University Ethics Sub-Committee (SMEC_2018-19_028).

Sixty seven percent of the study population consumed less than $400 \mathrm{mg}$ of choline; the recommended amount set by the European Food Safety Authority. There was a negative correlation between choline intake and anxiety ( $\mathrm{r}=-0.421, p=0.009$, Spearman's correlation). The mean anxiety score for females was $9.9 \pm 0.8$ and males $5.3 \pm 0.7$ ( $p=0.002$, Mann-Whitney $\mathrm{U}$ test). Within the abnormal anxiety group, the mean anxiety scores were higher in carriers of the rs4646343 risk allele compared to those homozygous for the G allele (GT+TT $13.8 \pm 2.3$ vs GG 11.5 $\pm 1.2, \mathrm{p}=0.018$, Mann-Whitney U test).

PEMT gene and choline intake may play a role in anxiety levels, however, further research in larger populations is needed to elucidate this relationship.

1. NHS Digital. (2016) [Available at: https://digital.nhs.uk/data-and-information/publications/statistical/adult-psychiatric-morbidity-survey/adultpsychiatric-morbidity-survey-survey-of-mental-health-and-wellbeing-england-2014]

2. Lai JS, Hiles S, Bisquera A et al. (2014) Am J Clin Nutr 99, 181-97

3. Bjelland I, Tell GS, Vollset SE et al. (2009) Am J Clin Nutr 90, 1056-1060

4. Zeisel SH, da Costa K-A (2009) Nutr Rev 67, 615-623 